\title{
Second-quarter biotech job picture
}

\author{
A quarterly snapshot of job expansions, reductions and availability in the biotech and pharma sectors.
}

T he second quarter of 2020 saw several pharma company expansions. In April, Baxter International said it would hire up to 2,000 employees globally, including 800 in the United States, in response to greater demand for medical devices due to the coronavirus pandemic.

In June, Fujifilm Diosynth announced a \$928 million investment to double biologics production capacity at its Hillerød, Denmark site by 2023 . The move comes a year after the contract development and manufacturing arm of the Japanese conglomerate acquired

\section{Table 1 | Who's hiring? Advertised openings at the 25 largest biotech companies}

\begin{tabular}{|c|c|c|c|c|}
\hline \multirow[t]{2}{*}{ Company ${ }^{a}$} & \multirow{2}{*}{$\begin{array}{l}\text { Number of } \\
\text { employees }\end{array}$} & \multicolumn{3}{|c|}{ Number of advertised openings } \\
\hline & & Monster & Linkedln & Biospace \\
\hline Amgen & 21,500 & 75 & 184 & 152 \\
\hline Gilead Sciences & 11,800 & 6 & 332 & 149 \\
\hline bioMérieux & 11,200 & 29 & 17 & 0 \\
\hline Biocon & 10,000 & 14 & 532 & 0 \\
\hline Celgene & 8,852 & 180 & 0 & 165 \\
\hline IDEXX Laboratories & 8,377 & 45 & 71 & 0 \\
\hline Biogen & 7,800 & 20 & 491 & 0 \\
\hline Regeneron Pharmaceuticals & 7,400 & 43 & 359 & 403 \\
\hline Illumina & 7,300 & 166 & 124 & 0 \\
\hline Novozymes & 6,279 & 0 & 2 & 0 \\
\hline Opko Health & 5,690 & 0 & 0 & 0 \\
\hline Endo Pharmaceuticals & 2,910 & 135 & 28 & 0 \\
\hline BioMarin Pharmaceutical & 2,849 & 29 & 116 & 0 \\
\hline BGI Genomics & 2,846 & 12 & 0 & 0 \\
\hline Alexion Pharmaceuticals & 2,656 & 3 & 61 & 0 \\
\hline GenScript Biotech & 2,620 & 0 & 0 & 8 \\
\hline Evotec & 2,617 & 0 & 4 & 22 \\
\hline Vertex Pharmaceuticals & 2,500 & 161 & 149 & 0 \\
\hline Myriad Genetics & 2,400 & 34 & 97 & 0 \\
\hline Alkermes & 2,300 & 55 & 62 & 0 \\
\hline Amphastar Pharmaceuticals & 2,078 & 0 & 7 & 0 \\
\hline BeiGene & 2,070 & 0 & 93 & 0 \\
\hline CK Life Sciences & 1,785 & 0 & 0 & 0 \\
\hline Emergent BioSolutions & 1,705 & 68 & 185 & 185 \\
\hline Biotest & 1,663 & 0 & 0 & 0 \\
\hline Total & & 1,075 & 2,914 & 1,084 \\
\hline
\end{tabular}

${ }^{a}$ As defined in Nature Biotechnology's survey of public companies $(36,576-584,2018)$. ${ }^{\text {A As searched }}$ on Monster.com, Linkedln.com and Biospace.com, 6 July 2020. Jobs may overlap. the former Biogen facility for $\$ 890$ million. The current buildout includes adding six mammalian cell bioreactors, installing a fill/finish production line with an expected capacity of 35 million units per year, and setting up a new packaging line for autoinjectors with automatic labeling. The site has already received a commitment from the COVID-19 Therapeutics Accelerator, which has reserved future manufacturing capacity.

On the negative side, Sage Therapeutics announced a major restructuring following the failure of a phase 3 study. In April, the biotech laid off 340 employees, slightly more than half its workforce.

And in June, Sanofi said it would eliminate 1,700 European jobs, including 1,000 in France, as part of a $\$ 1.8$ billion cost cutting over the next two years. It also announced it would invest $\$ 440$ million to build a new vaccine production site in France that would create 200 jobs.

Advertised biotechnology and pharmaceutical sector jobs in the job databases tracked by Nature Biotechnology were down in the second quarter of 2020 (Tables 1 and 2) compared with the previous quarter (Nat. Biotechnol. 38, 651, 2020).

Table 2 | Advertised job openings at the ten largest pharma companies

\begin{tabular}{lcccc} 
Company $^{\mathbf{a}}$ & $\begin{array}{l}\text { Number of } \\
\text { employees }\end{array}$ & \multicolumn{3}{c}{ Number of advertised openings } \\
\cline { 3 - 5 } & & Monster & Linkedln & Biospace \\
\hline Johnson \& Johnson & 135,100 & 13 & 727 & 0 \\
\hline Bayer & 115,500 & 16 & 237 & 0 \\
\hline Novartis & 108,000 & 403 & 304 & 0 \\
\hline Sanofi & 104,230 & 7 & 382 & 0 \\
\hline Abbott Laboratories & 103,000 & 3 & 847 & 0 \\
\hline GlaxoSmithKline & 95,490 & 2 & 704 & 0 \\
\hline Roche & 94,440 & 2 & 450 & 0 \\
\hline Pfizer & 92,400 & 4 & 309 & 0 \\
\hline Sinopharm & 69,720 & 0 & 0 & 0 \\
\hline Merck \& Co. & 69,000 & 1 & 361 & 0 \\
\hline Total & & 451 & 4,315 & 0 \\
\hline
\end{tabular}

a Data obtained from Statista. ${ }^{b}$ As searched on Monster.com, Linkedln.com and Biospace.com, 6 July 2020. Jobs may overlap.

Michael Francisco

Senior Editor, Nature Biotechnology.

Published online: 5 August 2020

https://doi.org/10.1038/s41587-020-0622-0 